ACT A U N IVERS I T A T IS L O D Z IENS IS

FOLIA LITTERARIA ROMANICA 11, 2016

http://dx.doi.org/10.18778/1505-9065.11.07

Inès Ben Zaïed

Université de Tunis

inesbenza@gmail.com

\title{
L'HYPOTYPOSE DANS LES NOUVELLES HISTOIRES TRAGIQUES DE BÉNIGNE POISSENOT
}

"Hypotyposis in Nouvelles Histoires tragiques of Bénigne Poissenot"

Summary - In Nouvelles Histoires tragiques by Bénigne Poissenot, the frequency of hypotyposis helps to arouse different emotions in the reader's heart. Indeed, quality of observation, painstaking precision, and the wealth of details drawn from "reality" impart to the narrative its full value. The author makes use of hypotyposis to create a "reality effect" which makes images more concrete and the scene more lively. His staging of human cruelty and the vicissitudes of fortune partakes of the dynamics of the text and has an impact on the reader's imagination. Finally, the main interest of this figure of style seems particularly linked to the author's both didactic and aesthetic project.

KEYWORDS - tragic story, Renaissance, description, morality, edification, truthfulness, emotion

RÉSUMÉ - Dans les Nouvelles Histoires tragiques de Bénigne Poissenot, la fréquence des hypotyposes vise à susciter, chez le lecteur, différentes émotions. En effet, la qualité de l'observation, le souci de précision et l'abondance des détails puisés dans la «réalité » confèrent au récit toute sa valeur. L'auteur exploite l'hypotypose afin de produire «un effet de réel» qui rend les images plus concrètes et la scène plus vivante. La mise en scène qu'il fait de la cruauté humaine et des vicissitudes de la Fortune participe de la dynamique du texte et agit sur l'imagination du lecteur. Enfin, l'intérêt principal de cette figure de style, nous semble particulièrement lié au projet didactique et esthétique de l'auteur.

MOTS-CLÉS - histoire tragique, Renaissance, description, morale, édification, véracité, émotion

L'histoire tragique se définit comme un récit bref véridique, à visée didactique et à tonalité pathétique. Elle marque, de ce fait, l'évolution que ce genre de narration connaît, dès la seconde moitié du XVI ${ }^{\mathrm{e}}$ siècle. En effet, le regard porté sur le crime, le goût du macabre et de l'horrible se développent énormément. Les auteurs optent pour le récit sanglant, exploitant la veine tragique qui était en vogue. Souvent, ils décrivent des batailles ou des scènes de combat et mettent l'accent sur les massacres et les martyrs que subissent les personnages.

Les Nouvelles Histoires tragiques de Bénigne Poissenot, publiées en 1586 et qui constituent le dernier recueil du genre au $\mathrm{XVI}^{\mathrm{e}}$ siècle, sont composées d' «histoires anciennes » et de «choses advenues de [son] temps». Pour le conteur, "l'histoire perdroit toute sa grace si les choses n'y estoyent représentées au vif et exprimées comme en un tableau, ainsi qu'elles se sont passées ${ }^{1}$. Vu les exigences esthétiques du genre, on assiste fréquemment à des scènes aussi violentes

${ }^{1}$ B. Poissenot, Nouvelles Histoires tragiques (désormais désignées par NHT), éd. J.-C. Arnould et R.A. Carr, Genève, Droz, 1996, « Prologue de l'auteur », p. 49. 
qu'émouvantes où Poissenot privilégie l'hypotypose afin d'agir sur l'imagination du lecteur et susciter, chez lui, plus d'émotion. Cette figure de suggestion visuelle regroupe l'ensemble de procédés permettant de rendre vivante une description au point que le lecteur « voit» le tableau se redessiner sous ses yeux.

Tout en s'interrogeant sur l'originalité de l'hypotypose et sa fonction dans le récit, on tentera, dans le cadre de cette étude, de rendre compte de la dimension visuelle de cette figure parfaitement exploitée par Poissenot. On s'intéressera, d'abord, à l'effet de réel produit, puis, à la mise en scène de la cruauté humaine et des vicissitudes de la Fortune. Enfin, on s'attachera à montrer la place importante qu'occupe l'hypotypose dans le projet esthétique et édifiant du conteur.

\section{Un effet de réel}

Pour répondre aux détracteurs du genre, Poissenot explique, dans le Prologue, sa théorie et révèle ses ressources esthétiques. Il n'hésite pas à évoquer certains reproches adressés contre l'histoire tragique dont le « langage affecté » représente « les choses si nuement qu'il semble que les voyons devant nos yeux, et non que les lisons $»^{2}$. L'auteur utilise « un argument de vraisemblance qui n'est pas sans rappeler le principe d'une rhétorique tridentine soucieuse d'utiliser l'énergie sensible des images ${ }^{3}$. Dans son recueil de nouvelles, il opte pour une représentation véritable, assurant une meilleure dénonciation des vices. Pour lui, «les vices y sont blasmez, on y loue la vertu, on appelle bois ce qui est bois, pomme ce qui est pomme; et n'y peut-on licitement desguiser ou masquer d'une fardée vertu ce qui n'est ny vertueux ny louable ${ }^{4}$.

À la recherche d'espaces plus vifs et plus majestueux, Poissenot emprunte un modèle rhétorique où le jeu complexe ne rompt pas avec la représentation concrète. Grâce à l'hypotypose, s'anime l'objet de la scène dont le déroulement s'effectue sous le regard du lecteur. Dans les Nouvelles Histoires tragiques, on remarque la fréquence de ce genre de figures dont la fonction n'est pas d'avancer l'action, mais plutôt d'actualiser la scène. D'ailleurs, même si l'hypotypose est une figure descriptive, il ne s'agit pas de simples ornements ou de détails surchargeant le récit.

La précision dont l'auteur fait preuve quand il donne une information, concerne aussi bien l'actualité que l'énoncé historique. Ce dernier n'hésitera pas à mener une enquête documentaire pour relater des événements historiques, comme la Saint-Barthélemy ou le tumulte du Bassigny, par exemple : «Si tu veux veoir plus amplement comme ce tumulte fut assopi, lis un petit traité qui en a esté faict

\footnotetext{
${ }^{2}$ NHT, « Prologue de l'auteur », ibid.

${ }^{3}$ T. Pech, Conter le crime. Droit et littérature sous la Contre-Réforme : Les histoires tragiques (1559-1644), Paris, Honoré Champion, 2000, p. 95.

${ }^{4} N H T$, «Prologue de l'auteur », p. 49.
} 
intitulé, le tumulte du Bassigny $»^{5}$. Le paradigme de la vue semble bien développé et parcourt tout le recueil afin de guider le lecteur. Le conteur mise sur la vigilance de ce dernier et sur sa qualité d'observateur, en le prenant à témoin. La redondance du verbe de perception « voir » est très significative, puisqu'elle vient confirmer l'importance du témoignage et l'authenticité du propos. L'hypotypose permet à l'auditeur de visualiser les choses, comme s'il s'agissait d'un tableau, d'une scène vivante se déroulant sous ses yeux ; ce qui justifie le rapprochement effectué entre l'image et le texte. Pour Thierry Pech, «l'image ne vaut pas en soi, mais pour ce qu'elle signifie, représente ou désigne $»^{6}$.

À la manière d'un chroniqueur, le conteur opte pour l'observation afin de décrire la scène et projeter le lecteur dans un monde qui lui est familier. Souvent, il enrichit ses tableaux de détails visuels qui animent la scène. Tel est le cas de la cinquième histoire tragique où le narrateur rend compte d'une réalité immédiate, celle du désastre et de l'épouvante, à travers la description détaillée de la ville de Montpellier :

La ville de Montpellier [...] estoit bien, avant les desolations qui luy sont survenues pour avoir changé de religion et espousé le parti des Protestans qu'elle tient encores pour le jourdhuy, une des plus belles, plaisantes et delicieuses villes de la Gaulle Narbonnoise ; elle a beaucoup diminué de son lustre maintenant, le siege qu'elle a soustenu ayant apporté la ruine et demolition de beaucoup de bastimens qui, edifiez aux lieux les plus plaisans d'alentour, et revestus de beaucoup d'arbres fruitiers et infinis beaux bocages, la rendoient d'autant plus illustre qu'elle apparoist aujourd'huy, par le spectacle des vestiges de ce qui plus servoit à son embellissement, ruineuse et desolée ${ }^{7}$.

Il s'agit d'une forme de description dont l'exactitude et la perfection tentent de peindre la vie. Le superlatif, ainsi que l'énumération des adjectifs «belles, plaisantes et delicieuses », mettent l'accent sur l'extrême beauté de la ville et accélèrent la chute de la phrase qui se termine par les expressions à connotation dévalorisante «ruineuse et desolée ». L'opposition entre le présent et l'imparfait de l'indicatif attire l'attention du lecteur sur le contraste entre l'état de la ville avant et après son envahissement par les protestants. En outre, l'expression métaphorique «le spectacle des vestiges », évoquant l'idée de ruines et de destruction, vise à sensibiliser davantage le lecteur aux désastres causés par les guerres de religion :

À peine avoient bien repris leur haleine les habitans, se rafreschissans des travaux qu'ils avoient soufferts, repoussans à leur pouvoir l'effort des assiegeans, que la peste se meit si bien en la ville qu'ils mouroient dru et menu comme pluie ; et fust l'air tellement infecté que les oiseaux mesmes, qui volloient par dessus le pourpris de la ville et la ceinture des murailles, tomboient à terre tous morts ${ }^{8}$.

\footnotetext{
${ }^{5} N H T, \mathrm{n}^{\mathrm{o}} 6, \mathrm{p} .251$.

${ }^{6}$ T. Pech, op. cit., p. 221.

${ }^{7} N H T, \mathrm{n}^{0} 5$, p. 213-214.

${ }^{8}$ Ibid., p. 214.
} 
Toutes ces images illustrent le climat apocalyptique associé au conflit opposant Catholiques et Protestants. Ainsi, on perçoit différents tableaux qui révèlent la vivacité de l'observation et l'intérêt accordé aux détails concrets. L'hypotypose offre la scène au lecteur, en la mettant sous ses yeux, afin de lui donner l'illusion d'y assister. Il convient de rappeler que la forme brève du récit s'adapte parfaitement à cet aspect, d'autant plus que le sens du mot «nouvelle » évoque l'idée d'une réalité proche ${ }^{9}$. Ainsi, la dernière histoire tragique du recueil confirme l'intérêt accordé aux faits contemporains. Ne correspondant à aucune source, elle constitue «le récit le plus personnel et le plus signifiant idéologiquement ${ }^{10}$. L'auteur se réfère à son expérience personnelle, ainsi qu'à ses souvenirs d'enfance pour faire le récit de « la sainte et louable vie » de son précepteur, Maitre George Pelleteret. En effet, l'histoire racontée est une histoire vraie ${ }^{11}$, mais Poissenot vise surtout à la rendre visible. Étant donné les circonstances de l'action, les lieux et les temps, ce récit peut être considéré comme un témoignage sur l'actualité. «S'inspirant à n'en pas douter du compte rendu judiciaire et du plaidoyer, Poissenot opère un brouillage important entre la représentation du vrai (du véridique) et celle de la fiction ${ }^{12}$.

En parlant d'Aristote et de sa théorie de la tragédie, Paul Ricœur évoque un aspect qu'il considère comme valable pour tout récit :

Le poète, en composant une fable, une intrigue, un mythos, offre une mimèsis, une imitation créatrice des hommes agissants. De la même manière, une logique des possibles narratifs, à quoi peut prétendre une analyse formelle des codes narratifs, ne s'achève que dans la fonction mimétique par laquelle le récit refait le monde humain de l'action ${ }^{13}$.

Aristote fait de la mimèsis le fondement même de sa Poétique. Il s'agit de mettre en valeur le pouvoir d'illusion de la littérature et des arts en général, quant

\footnotetext{
9 «Dans la logique de sa dénomination (féminin d'un adjectif si courant), la nouvelle fait référence à des réalités proches et familières en leur cadre», G.-A. Pérouse, «Des Nouvelles vrayes comme Evangile », in : La Nouvelle. Définitions, transformations, sous la dir. de B. Alluin et F. Suard, Université Charles de Gaulle-Lille III, Presses universitaires de Lille, 1990, p. 91-92.

${ }^{10}$ J.-C. Arnould, «Ordre et désordre dans quelques recueils narratifs de la seconde moitié du $\mathrm{XVI}^{\mathrm{e}}$ siècle (Boaistuau, Belleforest, Yver, Poissenot, Habanc) », in: Ordre et désordre dans la civilisation de la Renaissance, Actes du colloque RHR de Nice, septembre 1993, Publications de l’Université de Saint-Étienne, 1996, p. 320.

${ }^{11}$ Le conteur «narre là une biographie bien réelle, que nombre de témoins pouvaient contrôler », G.-A. Pérouse, Nouvelles françaises du XVI siècle. Images de la vie du temps, Genève, Droz, 1977, p. 291, note 78.

${ }^{12}$ F. Paré, «Formes du récit dans les Nouvelles histoires tragiques de Bénigne Poissenot », in: Écrire et conter, Mélanges de rhétorique et d'histoire littéraire du XVI ${ }^{\mathrm{e}}$ siècle offerts à Jean-Claude Moisan, Textes rassemblés et édités par M.-C. Malenfant et S. Vervacke, Québec, Presses de l'Université Laval, 2003, p. 99.

${ }^{13} \mathrm{P}$. Ricœur, «Expliquer et comprendre. Sur quelques connexions remarquables entre la théorie du texte, la théorie de l'action et la théorie de l'histoire », Revue Philosophique de Louvain, Quatrième série, t. 75, n ${ }^{\circ}$ 25, 1977, p. 132-133.
} 
à la représentation du réel. Ainsi, si l'art est une imitation, selon la poétique d'Aristote, on s'interroge sur le degré de réalisme de cette mimèsis chez Poissenot.

Certes, «les théoriciens de la Renaissance et du XVII ${ }^{\mathrm{e}}$ siècle placeront presque tous l'imitation des choses au cœur de leur esthétique. Si les uns lui conservent (non sans difficulté logique) sa double face - copie conforme et quête du vraisemblable -, les autres opèrent un choix : l'art comme illusion ou l'art comme révélation de l'être en puissance ${ }^{14}$. Pour notre conteur, l'écriture n'est autre qu'un spectre de la vérité ; elle reflète, comme dans un miroir, la misère de l'homme, la précarité du bonheur et les caprices de la fortune: "Qui nous enseignera cela, de qui l'apprendrons-nous si ce n'est de l'histoire, qui nous la représente comme dedans un miroir ? ${ }^{15}$

En observant attentivement ce qui est vrai, l'auteur incite son lecteur, non seulement à imaginer la scène mais à la revivre «réellement », en la visualisant, grâce à l'hypotypose. Dessins, courbes et couleurs animent ces récits et leur donnent plus de richesse et de perfection. Certes, imiter la réalité, c'est embrasser son cadre et ses formes les plus visibles. Gérard Genette établit une opposition entre la mimèsis («ce qui donne à voir ») et la diegesis (« ce qui est raconté ») $)^{16}$. Pour Roland Barthes, l' «effet de réel», constitue le «fondement de ce vraisemblable inavoué qui forme l'esthétique de toutes les œuvres courantes de la modernité ». En fait, la notion de représentation ne vise pas à reproduire la réalité, mais à la transformer, à la transposer, en assurant le caractère «vraisemblable » de la fiction représentée ; car «supprimé de l'énonciation réaliste à titre de signifié de dénotation, 'le réel' y revient à titre de signifié de connotation ${ }^{17}$. Pour donner l'illusion du « vrai », Poissenot tente de capturer le réel, dans son aspect le plus concret, mais aussi le plus étendu. Son but étant d'élargir le champ littéraire, il rompt les barrières entre « réalité » et « fiction».

L'hypotypose répond au besoin de l'auteur de produire un «effet de réel », lui permettant de donner une image plus ou moins «fidèle » du monde et de la condition humaine. Cependant, il n'est pas question d'une simple image, mais d'un tableau mouvant, d'une réalité vivante qui se déroule sous les yeux du lecteur / spectateur. Afin de cultiver l'illusion «mimétique » où le fictif fusionne avec le réel, le narrateur joue sur le côté spectaculaire de la scène.

\footnotetext{
${ }^{14}$ M. Jeanneret, «Les Tragiques : mimèsis et intertexte », in : Le Signe et le texte. Études sur l'écriture au XVI siècle en France, éd. L. D. Kritzman, Lexington, U.S.A., French Forum, 1990, p. 107.

${ }^{15}$ NHT, « Prologue de l'auteur », p. 58.

${ }^{16}$ Voir G. Genette, Figures II, Paris, Seuil, 1966 et Figures III, Paris, Seuil, 1969.

${ }^{17}$ R. Barthes, "L'effet de réel », Communications, $\mathrm{n}^{\mathrm{o}} 11$, Recherches sémiologiques. Le vraisemblable, Paris, Seuil, 1968, p. 88.
} 


\section{Mise en scène de la cruauté humaine et des vicissitudes de la fortune}

Dans les Nouvelles histoires tragiques, on assiste au passage du simplement descriptif à la mise en scène qui donne au texte plus de vigueur et de vitalité ; ce qui permet de privilégier le mouvement et le dynamisme, en rendant les mots « visibles », en métamorphosant les images. L'hypotypose permet de peindre une scène vivante qui se transforme en véritable spectacle, dans le but de surprendre le lecteur et frapper son attention. Ainsi, on peut dire que le recours à cette figure descriptive alimente la représentation de la violence, qui semble en harmonie avec le goût du récit tragique pour les scènes sanglantes et les morts atroces. La sixième histoire tragique relate le massacre de Maître Pelleteret, lâchement assassiné par un groupe de reîtres dont il dénonce le comportement inhumain :

un de la troupe luy donna un coup de pistolle par la teste et le renversa par terre. Le pensant estre mort, ils poursuivirent leur chemin, le laissans gisant de son long sur terre. Guieres ne furent esloignez qu'il se leva, bien qu'il fust blessé à mort, et courut tant que ses pieds le pouvoient porter vers son village, mais les meurdriers l'ayans apperceu tournerent bride, et à grands coups d'estoc, le vindrent achever, luy traversant le corps de part en part $^{18}$.

L'auteur brosse ici un tableau bien détaillé où l'on perçoit la cruauté de la scène et son caractère sanglant. Il véhicule un message précis contre les Huguenots, en insistant sur leur trahison. On ne peut nier la sincérité d'un tel témoignage, d'autant plus que la victime de ce crime n'est autre que l'ancien précepteur du conteur, qui prend part personnelle au drame. L'accumulation des verbes d'action et le grossissement des détails mettent l'accent sur la brutalité des actes commis par ces criminels. Pour donner à voir l'action et la rendre plus dramatique, l'écrivain utilise le discours direct: «Meurdriers du sang racheté par l'effusion du sang du fils de Dieu, Jesus-Christ, juste juge, jugera entre vous et moy, et ne laissera voz inquitez impunies ${ }^{19}$. La réplique du prêtre mourant est considérée par Poissenot comme «parolle Prophetique $»^{20}$. Face à une telle scène, le lecteur ne peut cacher son émotion, voire son indignation.

D'autres tableaux de ce genre s'offrent au regard. Dans les Nouvelles Histoires tragiques se multiplient les scènes de crimes. Sous la menace d'un mariage forcé, Eliude et Floridanus, héros de la première histoire tragique, se trouvent dans l'obligation de fuir. L'amoureux est attaqué par des bandits ; il était sur le point de vaincre ses agresseurs, quand, soudain, il trébuche. Le narrateur met l'accent sur les moindres détails de la scène illustrant la défaite inattendue du héros qui va engendrer sa mort : «Ce qu'advisant Floridanus, eschauffé comme un taureau qui combat pour sa genisse, il se jetta après eux tant indiscrettement qu'il se laissa tomber de son long à travers la porte, et perdit par ce moyen la

\footnotetext{
${ }^{18}$ NHT, n ${ }^{\mathrm{o}} 6$, p. 250.

${ }^{19}$ Ibid.

${ }^{20}$ Ibid.
} 
victoire qui estoit jà entre ses mains $»^{21}$. Les verbes d'action, ainsi que la comparaison «comme un taureau qui combat pour sa genisse», restituent l'image et mettent l'accent sur l'ardeur qui anime le personnage. La mise en scène du mouvement traduit ici les vicissitudes de la Fortune face à laquelle l'homme demeure impuissant. L'emploi du passé simple souligne la rapidité de l'action et annonce la chute accidentelle, entrainant la perte de Floridanus. Poissenot semble marqué par tout ce qui relève du changement brusque et imprévu ${ }^{22}$ :

Il n'y a chose plus apte et convenable, pour la delectation du lecteur, que la variété des temps et les diverses mutations de fortune, lesquelles, combien qu'elles ayent esté de peu de plaisir à ceux qui les ont experimentées et souffertes, si est-ce qu'elles sont plaisantes et agreables à lire.

Ce qui le distingue des autres conteurs du XVI ${ }^{\mathrm{e}}$ siècle dont il est le disciple, c'est sa réflexion sur la vanité de l'existence humaine. À travers ce genre de scènes aussi vivantes que mouvementées, l'auteur cherche à produire chez le lecteur une impression. Il exprime, de ce fait, la nécessité de tenir ce dernier en haleine. Il attise sa curiosité et cultive son imagination, en garantissant l'effet de surprise ; l'attente crée le suspense, en retardant le récit :

Il s'agit, par un procédé d'imitation, de précipiter l'émotion de l'allocuteur, d'induire en lui un effet de sidération, qui le met comme bouche bée, devant une représentation si forte qu'elle s'impose à lui, au-delà (en deçà) de la narration, comme la seule réalité, une réalité à laquelle il assiste passivement, en spectateur impuissant, mais fasciné. L'hypotypose est de l'ordre de la stupéfaction, tant dans le discours que dans l'action : elle étonne - au sens classique du terme - et laisse pétrifiééc.

Dans ce type de narration où abondent les plaintes et les lamentations, l'émotion semble privilégiée. D'ailleurs, le choix de sujets pathétiques et dramatiques répond parfaitement au schéma narratif de l'histoire tragique. N'éprouvant pas le besoin d'étoffer son texte d'ornements excessifs ou inutiles, Poissenot réduit la sixième histoire à quelques pages. Ce récit poignant mettant l'accent sur le martyre que souffrait Maître Pelleteret, suscite la pitié du lecteur et son indignation face à de tels comportements barbares. D'ailleurs, ce dernier semble bien préparé à ce genre de scènes "piteuses » : «les inconveniens qui en surviennent ne te pourroient estre mieux descrits que l'histoire presente te les peindra, digne d'autant de pitié et commiseration que le faict en est deplorable $»^{24}$.

L'auteur s'intéresse énormément à la réception de son œuvre. Dans son Prologue, il énumère les différentes émotions que suscite la lecture d'un récit tragique chez le lecteur :

\footnotetext{
${ }^{21} N H T, \mathrm{n}^{\mathrm{o}} 1, \mathrm{p} .115$.

${ }^{22} N H T$, «Prologue de l'auteur », p. 46.

${ }^{23}$ Y. Le Bozec, «L'hypotypose : un essai de définition formelle », L'Information Grammaticale, $\mathrm{n}^{\mathrm{o}} 92,2002$, p. 5.

${ }^{24}$ NHT, n ${ }^{\circ} 5$, p. 213.
} 
Mais les accidens et diverses fortunes d'un homme excellent et renommé remplissent le lecteur d'admiration, d'attente, de joye, de facherie, d'esperance, de crainte; et si l'issue en est bonne et notable, lors l'esprit du lecteur est remply d'une volupté grande par ceste joyeuse et plaisante lecture $^{25}$.

Dans son argumentation, il s'appuie sur Cicéron et ses Épîtres familières, traduites par Belleforest. Certes, ce genre de récits éveille chez le lecteur les sentiments et les émotions les plus contrastés : gaieté et affliction, plaisir et pitié, espérance et crainte... Poissenot insiste sur la diversité des émotions, réclamée par Cicéron ; néanmoins, le champ lexical de la joie («volupté », «joyeuse », «plaisante ») révèle que la lecture du livre n'est autre qu'un parcours qui se fait dans l'euphorie et la bonne humeur. Il est donc clair que la délectation repose sur l'alternance des émotions ; Thierry Pech parle même de «la délectation tragique de Poissenot $»^{26}$. La lecture de ces récits offre un «admirable contentement d'esprit $\gg^{27}$, selon l'expression du conteur. Elle acquiert une fonction thérapeutique, puisqu'elle assure soulagement et réconfort au lecteur.

Certes, «l'hypotypose doit toucher les sens et non la raison, elle doit faire appel au plaisir et à l'épouvante, friser l'insoutenable en une jouissance intense mais terrible $\gg^{28}$. Dans son Prologue, l'auteur fait allusion à un passage du $D e$ natura rerum de Lucrèce ; il s'agit du $2^{\mathrm{e}}$ chant « Suave mari magno... » :

[...] l'asseurée souvenance de la douleur passée a je ne sçay quelle delectation en soy, pleine d'une volupté et plaisir incroyable pour celuy qui en est eschappé, et qui peut grandement retenir les esprits des hommes en lisant telle chose. Tout ainsi que quand d'un port nous voyons quelque navire ou vaisseau perir en haute mer, nous nous sentons en mesme heure esmeus diversement, estans et joyeux et fachez tout d'un coup : joyeux, pour ne participer au naufrage de ceux qui devant nos yeux descendent au Royaume de Neptune ; fachez de l'inconvenient et malheur que les voyons, sans leur pouvoir donner secours et les soulager, ainsi que volontiers desirerions s'il estoit en nostre puissance de ce faire ${ }^{29}$.

Cette scène, cadre d'une peinture vivante, frappe l'attention du lecteur. On remarque la répétition du verbe « voir » et l'emploi de l'expression «devant nos yeux ». L'emploi de termes précis vise à offrir au lecteur une perspective plus claire et plus palpable, lui permettant d'imaginer la scène. Les images servent à décrire la descente au «Royaume de Neptune » pour mieux traduire l'idée de danger. Le naufrage dont il est question ici suggère le déclin, la finitude et l'achèvement.

La vue d'un tel spectacle suscite les émotions les plus diverses (« esmeus diversement »). Le lecteur est tiraillé entre deux sentiments contradictoires. Tout

\footnotetext{
${ }^{25}$ NHT, « Prologue de l'auteur », p. 46.

${ }^{26}$ Th. Pech, op. cit., p. 91 sq.

${ }^{27}$ NHT, « Prologue de l'auteur », p. 47.

${ }^{28}$ Y. Le Bozec, op. cit., p. 5.

${ }^{29}$ NHT, « Prologue de l'auteur », p. 45.
} 
en se réjouissant d'être à l'abri du danger, il fait preuve de compassion à l'égard de ceux qui ont péri. Plaisir et contrariété se trouvent associés. En fait c'est en voyant les maux d'autrui qu'on parviendrait à oublier ses propres malheurs.

L'hypotypose met l'accent sur le caractère vif et rapide de l'action. La précision visuelle que l'on perçoit dans l'ensemble du recueil donne l'illusion au lecteur d'être devant un fragment de vie, réel et effectif. L'image devient animée et la scène est renouvelée, revisitée, voire «re-configurée ». Ce procédé littéraire amplifie la violence représentée, qui suscite à la fois la curiosité et la compassion du lecteur. Générer l'émotion constitue un point essentiel dans l'esthétique de l'histoire tragique ${ }^{30}$. Notre conteur, considéré comme le théoricien du genre, insiste énormément sur cet aspect qu'il rattache également au projet d'édification du lecteur. Quel rapport y a-t-il entre le choix de l'hypotypose et le projet didactique de Poissenot?

\section{L'hypotypose et le projet de Poissenot}

La dimension didactique de l'histoire tragique, souvent rapprochée de l'exemplum, est essentiellement liée au désir d'enseigner une morale, de servir la mémoire collective. Elle se traduit particulièrement, chez Poissenot, par la fréquence des sentences et des proverbes, ainsi que par les dénouements tragiques qui émeuvent le lecteur et l'incitent à la réflexion. L'auteur inscrit la sixième histoire tragique dans un contexte historique sanglant qui semble en harmonie avec la fin tragique de l'histoire, ainsi qu'avec la visée morale et esthétique du genre. En effet, le récit s'achève avec la mort de Maître Pelleteret, mais aussi avec le châtiment des coupables et le rétablissement de l'ordre.

En outre, l'enseignement moral est également assuré grâce à la portée véridique de ce genre de narration ${ }^{31}$. Le narrateur exprime le besoin d'accréditer son propos et de garantir sa sincérité, en précisant l'origine de l'histoire et en certifiant l'authenticité des récits. Ainsi, «si la nouvelle s'affirme si hautement véridique, c'est aussi [...] parce que la parole qu'elle est veut être comprise, méditée, vécue $»^{32}$. Le goût pour la vérité permettrait de donner un air de réalité à l'histoire

\footnotetext{
${ }^{30}$ « Par opposition à la nouvelle facétieuse, pouvant se contenter de vraisemblance pour faire rire, la nouvelle tragique a besoin de la vérité afin d'assurer à la pitié le statut d'une expérience authentique. La réception intellectuelle de l'histoire, passant par les structures du langage, devient ainsi la condition de la réaction affective du lecteur », W.K. Pietrzak, Le Tragique dans les nouvelles exemplaires en France au XVI $I^{e}$ siècle, Łódź, Wydawnictwo Uniwersytetu Łódzkiego, 2006, p. 44.

31 «Pour Poissenot, la vérité de la représentation est destinée à faire tenir les vices en horreur et sans elle, l'édification n'opérerait pas. », J.-C. Arnould, « Des paroles honnêtes dans les histoires tragiques (Taillemont, Boaistuau, Belleforest, Habanc, Poissenot)», in : La Politesse amoureuse de Marsile Ficin à Madeleine de Scudéry. Idées, codes, représentations, dir. F. Greiner et J.-C. Ternaux, Franco-Italica, ${ }^{\circ} 15,1999$, p. 365.

${ }^{32}$ G.-A. Pérouse, « Des Nouvelles vrayes comme Evangile », op. cit., p. 94.
} 
racontée, la rendant plus familière. Poissonot puise ses sujets, soit dans l'Histoire, soit dans l'actualité, à la manière des canards d'information et des chroniques judiciaires. De ce fait, le recours à la réalité historique peut être considéré comme un procédé permettant au narrateur d'authentifier son discours.

Tout en optant pour l'histoire tragique, l'auteur inscrit le récit dans un contexte historique bien déterminé. Il accorde une place privilégiée, dans son projet didactique, à l'Histoire. Déjà, dans son Prologue, il explique l'utilité de cette dernière, permettant de restaurer les valeurs sociales et morales révolues. S'agitil, dans ce cas, de récupérer un passé perdu ou de restituer l'Histoire et de la recréer, sous le regard du lecteur?

Si le discours véridique adopté par les conteurs du $\mathrm{XVI}^{\mathrm{e}}$ siècle rapproche le genre de l'Histoire, Pech parle surtout d'une rhétorique du « vrai historique $»^{33}$. Étant frappé par la violence des guerres civiles, Poissenot choisit un thème qui a une valeur actuelle. Mais ce thème, appuyé par le recours récurrent à l'hypotypose, ne pourrait passer seulement pour un simple élément de vraisemblance ou de vérité historique. Il rend compte également du désordre social, religieux et moral qui régit le pays. Évoquer les temps de guerre, avec autant de précision et d'exactitude, est, pour le conteur, l'occasion de mimer le chaos qui bouleverse le monde. Il s'agit bien de la vision de l'auteur dont le souci est de réagir contre la décadence. C'est pour cela qu'il nous incite à nous inspirer des expériences de nos prédécesseurs pour en tirer profit :

Puis qu'elle est la messagere de l'antiquité, il ne se peut faire qu'elle ne soit comme une certaine boutique, en laquelle on trouve la manière et façon de vivre honnestement en ce monde; de laquelle aussi on peut, en choses douteuses, prendre conseils certains et infallibles, mesurant ses actions selon que l'on verra qu'il aura bien ou mal pris aux autres en mesme cas ${ }^{34}$.

Nul ne peut nier la richesse de l'Histoire, considérée comme la meilleure source où les écrivains puisent leurs sujets. La comparaison « comme une certaine boutique » fait de ces histoires de véritables témoignages authentiques et efficaces. Loin de se réduire à un procédé d'écriture, l'hypotypose sous-tend le projet didactique de notre conteur, fixé au préalable dans son Prologue :

On tire d'icelle des patrons, pourtraits et images qu'on se propose devant les yeux, pour en la contemplation de telles medales, apprendre à bien vivre, abhorrant le vice, et suyvant le chemin de vertu que ceux que lisons avoir esté vertueux nous ont monstré ${ }^{35}$.

La relative «qu'on se propose devant les yeux » fait allusion au principe même de l'hypotypose, qui constitue une étape primordiale de cette démonstration, fondée sur les expériences d'autrui. Les différents héros, comme Maître

\footnotetext{
${ }^{33}$ T. Pech, op. cit., p. 47.

${ }^{34}$ NHT, «Prologue de l'auteur », p. 54-55.

${ }^{35}$ Ibid., p. 55.
} 
Pelleteret $^{36}$, Alexandre le Grand ou Jules César ${ }^{37}$ sont, pour le conteur, des modèles à imiter. Le lecteur n'a qu'à suivre le parcours que lui propose le narrateur, en actualisant les mots qui redessinent la scène :

Parquoy prenant le sentier de la prairie, et tiré de mon inclination, qui se plaist à choses recreatives et delectables, je parvins en fin en un plaisant verger où, me veautrant sur l'herbe drue, et cueillant romarins, œillets, marjolaine, marguerites et autres fleurs, je n'en eu plustost ma main pleine que j'en façonnay un chapeau et guirlande, tissue de la façon que cy après la verras ${ }^{38}$.

L'usage de l'énumération permet d'exposer, de façon détaillée, les différents éléments qui constituent le tableau. Ce parcours riche et varié guidera le lecteur dans cette expérience édifiante, lui permettant d'accéder à une meilleure compréhension ou interprétation de l'œuvre. L'auteur puise, généralement, dans la rhétorique pour développer son point de vue et conférer plus d'ampleur à son texte. Son objectif, avant tout, est de plaire et de convaincre à la fois :

[... ] pour ce qu'elle conserve la mémoire des choses qui ont esté bien ou mal exploitées ; en partie aussi pour ce qu'elle advertit un chacun de son debvoir beaucoup mieux que ne sçauroient faire les stastues, pyramides, pourtraits et images de nos ancestres et devanciers ${ }^{39}$.

Cette description privilégie la peinture des faits, en produisant un effet de réel. L'hypotypose conditionne le pacte qui unit le narrateur au lecteur. Ce dernier est plongé au cœur d'une réalité sanglante, celle des guerres de religion qui ravagent le pays. Il s'agit de l'attitude d'un destinataire observateur dont le but est de voir et de tout savoir. De ce fait, l'auteur confirme une certaine complicité avec un lecteur curieux et éveillé, qui n'hésite pas à visualiser les différentes scènes et à juger lui-même de la situation. Il réactualise son discours, en offrant à ce dernier la possibilité d'interpréter le récit à sa manière : "Nous te faisons juge si le faict raconté en l'histoire suivante ne fut un trait de cruauté plus que barbaresque, dequoy, après l'avoir leue, tu diras ton advis si bon te semble ${ }^{40}$.

\footnotetext{
${ }^{36}$ L'héroïsme de Maître Pelleteret semble digne des grands héros des tragédies classiques du XVII ${ }^{\mathrm{e}}$ siècle. Cet homme de « saincte et louable vie » est un personnage exemplaire, un modèle de sagesse et de vertu : «Ceux qui auront esté lettrez et sçavans resplendiront comme la clarté et lueur du Firmament, et ceux là qui enseignent plusieurs, et leur monstrent le chemin pour etre droicturiers et vivre justement selon Dieu en ce monde, sont comme estoilles flamboyantes qui esclaireront à jamais » (NHT, $\mathrm{n}^{\circ} 6, \mathrm{p}$. 238). En mettant l'accent sur la grandeur du personnage, la métaphore de la lumière valorise Maître Pelleteret et sa mission.

${ }^{37}$ « Jules Cesar, le premier et le plus illustre de tous les Empereurs Romains, et que pouvons à bon droict non seulement comparer mais, qui plus est, preferer à Alexandre le grand, ne mourut en tant de sanglantes batailles [...] pour venir donner un triste spectacle de sa mort au peuple romain... », NHT, $\mathrm{n}^{\mathrm{O}} 2$, p. 128-129.

${ }^{38}$ NHT, «Prologue de l'auteur », p. 54.

${ }^{39}$ Ibid., p. 57.

${ }^{40} N H T, n^{\circ} 6$, p. 235.
} 
« Nous te faisons juge », précise le conteur, transformant le rôle de spectateur assigné au lecteur en rôle d'acteur. Jean-Claude Arnould parle d' « une participation active $\gg^{41}$ du lecteur qui l'inciterait à réfléchir. Ainsi, dans ce recueil de nouvelles, une simple anecdote peut devenir un grave moment de réflexion, voire de remise en question. Une vision pessimiste de l'homme et du monde ${ }^{42}$ se confirme dès le Prologue : «De mesme, lors que pensons estre en repos, qui coustumierement nous vient après le travail, nous experimentons les proverbes veritables qui nous admonestent qu'il n'y a en ce monde aucune felicité et beatitude perdurable $»^{43}$.

Bénigne Poissenot exploite ses qualités d'historien et adopte une approche «visualisante », truffée de détails réalistes. Ce « chroniqueur des faits de son époque $[\ldots]$ est surtout un pédagogue des lois de l'histoire ${ }^{44}$. L'hypotypose répond aux souhaits du conteur de dire vrai et de rester authentique, en renforçant le dynamisme exemplaire des récits. En effet, elle semble en harmonie avec le système de valeurs qu'il véhicule, permettant l'accomplissement de son projet édifiant. Elle garantit la variété d'impressions ${ }^{45}$ et confirme la fonction rédemptrice de la littérature.

\section{Conclusion}

L'hypotypose, figure suggestive par excellence, constitue une des techniques employées dans les Nouvelles Histoires tragiques pour mener le lecteur à une révélation, à travers la peinture de tableaux aussi concrets qu'émouvants. Mises à part les scènes de violence où elle joue un rôle fondamental, elle fait partie intégrante de tout un art du discours persuasif. En recherchant un effet de réel, Poissenot opte pour l'efficacité rhétorique et privilégie la dimension didactique de la représentation. L'intérêt accordé à tout ce qui est spectaculaire se traduit également chez ses prédécesseurs, en particulier Boaistuau et son Théâtre du monde ; il se poursuit au XVII ${ }^{\mathrm{e}}$ siècle, avec L'Amphithéâtre sanglant de JeanPierre Camus. D'ailleurs, pour ce dernier, «le monde est le sanglant amphithéâtre de semblables actions qui arrivent tous les jours devant nos yeux et qui

\footnotetext{
${ }^{41}$ J.-C. Arnould, «Sur la sixième des Nouvelles histoires tragiques de Bénigne Poissenot», in : Narrations brèves. Mélanges de littérature ancienne offerts à Krystyna Kasprzyk, Warszawa, Université de Varsovie, 1993, p. 206.

${ }^{42}$ Ce pessimisme fondamental qui marque la seconde moitié du XVI ${ }^{\mathrm{e}}$ siècle est associé à la misérable condition humaine; il se perçoit également chez François de Belleforest, le prédécesseur de Poissenot. Voir l'article de W.K. Pietrzak, «François de Belleforest et la crise de l'optimisme en France au $\mathrm{XVI}^{\mathrm{e}}$ siècle », in : Écrire la rupture, Actes du colloque international de Lyon, 16-17 septembre 2002, sous la dir. de C. Queffélec et R.-P. Colin, Tusson, Du Lérot, 2003, p. 161-174.

${ }^{43} N H T, \mathrm{n}^{\mathrm{o}} 2$, p. 123.

${ }^{44}$ F. Paré, op. cit., p. 103.

${ }^{45}$ Pitié / crainte, joie / larmes, incertitude / espérance...
} 
sont d'autant moins remarquées qu'elles nous sont plus familières ${ }^{46}$. Ces «peintures parlantes » offrent au lecteur un spectacle vivant, en permettant à ces conteurs de défendre un idéal et une visée esthétique.

Le regard que l'auteur porte sur le réel révèle à quel point le goût a changé en matière de narration brève, à la fin du siècle. On perçoit, chez lui, le désir de rendre hommage au monde, à la vie, dans toute sa splendeur, mais aussi dans toute sa complexité. Si le conteur préfère l'image animée et vivante au concept, c'est pour offrir au lecteur un aperçu riche et varié sur la société de son temps. Le choix de l'hypotypose comme figure alimente l'esthétique du conteur où se conjuguent le fictif et le vrai, le sublime et le banal, le subjectif et l'authentique, transformant l'acte de lire en expérience profonde et unique.

\section{Bibliographie}

Arnould, Jean-Claude, «Sur la sixième des Nouvelles histoires tragiques de Bénigne Poissenot », in: Narrations brèves. Mélanges de littérature ancienne offerts à Krystyna Kasprzyk, Warszawa, Université de Varsovie, 1993, p. 205-213

Arnould, Jean-Claude, «Ordre et désordre dans quelques recueils narratifs de la seconde moitié du XVI ${ }^{\mathrm{e}}$ siècle (Boaistuau, Belleforest, Yver, Poissenot, Habanc) », in : Ordre et désordre dans la civilisation de la Renaissance, Actes du colloque RHR de Nice, septembre 1993, Publications de l'Université de Saint-Étienne, 1996, p. 309-320

Arnould, Jean-Claude, «Des paroles honnêtes dans les histoires tragiques (Taillemont, Boaistuau, Belleforest, Habanc, Poissenot) », in : La Politesse amoureuse de Marsile Ficin à Madeleine de Scudéry. Idées, codes, représentations, dir. Franck Greiner et Jean-Claude Ternaux, Franco-Italica, $\mathrm{n}^{\mathrm{o}} 15,1999$, p. 359-367

Barthes, Roland, «L'effet de réel », Communications, $\mathrm{n}^{\circ} 11$, Recherches sémiologiques. Le vraisemblable, Paris, Seuil, 1968, p. 84-89

Camus, Jean-Pierre, L'Amphithéâtre sanglant, édité par Stéphan Ferrari, Paris, Honoré Champion, 2001

Jeanneret, Michel, «Les Tragiques : mimèsis et intertexte», in : Le Signe et le texte. Études sur l'écriture au XVI siècle en France, éd. Laurence D. Kritzman, Lexington, U.S.A., French Forum, 1990, p. 101-113

Le Bozec, Yves «L'hypotypose : un essai de définition formelle », L'Information Grammaticale, $n^{\circ} 92,2002$, p. 3-7

Paré, François, «Formes du récit dans les Nouvelles histoires tragiques de Bénigne Poissenot», in : Écrire et conter, Mélanges de rhétorique et d'histoire littéraire du XVI ${ }^{\mathrm{e}}$ siècle offerts à Jean-Claude Moisan, Textes rassemblés et édités par Marie-Claude Malenfant et Sabrina Vervacke, Québec, Presses de l'Université Laval, 2003, p. 97-108

Pech, Thierry, Conter le crime. Droit et littérature sous la Contre-Réforme : Les histoires tragiques (1559-1644), Paris, Honoré Champion, 2000

Pérouse, Gabriel-André, Nouvelles françaises du XVI siècle. Images de la vie du temps, Genève, Droz, 1977

Pérouse, Gabriel-André, «Des Nouvelles vrayes comme Evangile», in : La Nouvelle. Définitions, transformations, sous la dir. de Bernard Alluin et François Suard, Université Charles de Gaulle-Lille III, Presses universitaires de Lille, 1990, p. 89-99

${ }^{46}$ J.-P. Camus, L'Amphithéâtre sanglant, éd. S. Ferrari, Paris, Honoré Champion, 2001, «L'auteur au lecteur », p. 179. 
Piégay-Gros, Nathalie, Introduction à l'intertextualité, Paris, DUNOD, 1996

Pietrzak, Witold Konstanty, «François de Belleforest et la crise de l'optimisme en France au XVI ${ }^{\mathrm{e}}$ siècle », in: Écrire la rupture, Actes du colloque international de Lyon (16-17 septembre 2002), sous la dir. de Christine Queffélec et René-Pierre Colin, Tusson, Du Lérot, 2003, p. 161-174

Pietrzak, Witold Konstanty, Le Tragique dans les nouvelles exemplaires en France au XVI siècle, Łódź, Wydawnictwo Uniwersytetu Łódzkiego, 2006

Poissenot, Bénigne, Nouvelles Histoires tragiques, édition établie et annotée par Jean-Claude Arnould et Richard A. Carr, Genève, Droz, 1996

Ricœur, Paul, «Expliquer et comprendre. Sur quelques connexions remarquables entre la théorie du texte, la théorie de l'action et la théorie de l'histoire », Revue Philosophique de Louvain, Quatrième série, t. 75, nº 25, 1977, p. 126-147

\section{Inès Ben Zaïed}

Inès Ben Zaïed est enseignante à l'Université de Tunis. Elle est spécialiste de littérature française du XVI ${ }^{\mathrm{e}}$ siècle, titulaire d'un doctorat, préparé dans le cadre d'une cotutelle internationale de thèse, entre l'Université de la Manouba (Tunisie) et l'Université de Rouen (France). Ses principaux travaux portent sur la narration brève de la Renaissance, notamment sur la nouvelle exemplaire, la nouvelle facétieuse et l'histoire tragique. 